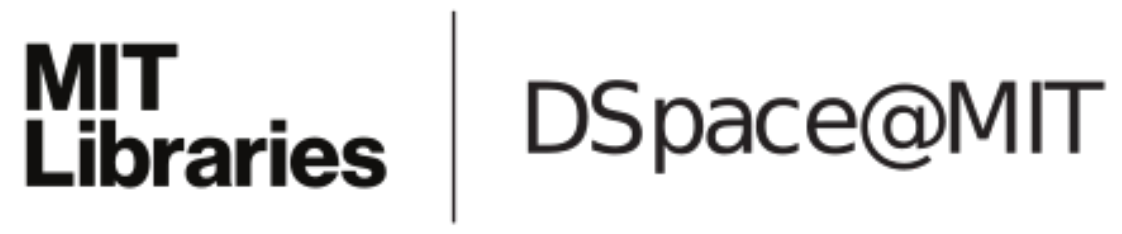

\author{
MIT Open Access Articles
}

Synthetic approaches to understanding biological constraints

The MIT Faculty has made this article openly available. Please share how this access benefits you. Your story matters.

Citation: Velenich, Andrea, and Jeff Gore. "Synthetic Approaches to Understanding Biological Constraints." Current Opinion in Chemical Biology 16, no. 3-4 (August 2012): 323-328.

As Published: http://dx.doi.org/10.1016/j.cbpa.2012.05.199

Publisher: Elsevier B.V.

Persistent URL: http://hdl.handle.net/1721.1/88494

Version: Author's final manuscript: final author's manuscript post peer review, without publisher's formatting or copy editing

Terms of use: Creative Commons Attribution-Noncommercial-Share Alike 


\title{
Synthetic approaches to understanding biological constraints
}

\author{
Andrea Velenich ${ }^{\mathrm{a}}$ and Jeff Gore ${ }^{\mathrm{a}}$ \\ aMassachusetts Institute of Technology, Department of Physics, Cambridge, MA, USA
}

\begin{abstract}
Microbes can be readily cultured and their genomes can be easily manipulated. For these reasons, laboratory systems of unicellular organisms are increasingly used to develop and test theories about biological constraints, which manifest themselves at different levels of biological organization, from optimal gene expression levels to complex individual and social behaviors. The quantitative description of biological constraints has recently advanced in several areas, such as the formulation of global laws governing the entire economy of a cell, the direct experimental measurement of the tradeoffs leading to optimal gene expression, the description of naturally occurring fitness landscapes, and the appreciation of the requirements for a stable bacterial ecosystem.
\end{abstract}

\section{Introduction}

Living organisms are optimized by evolution to survive and reproduce. Optimization targets all levels of biological organization at once, from elementary biomolecules to large anatomical features, from the expression levels of single genes to complex individual and social behaviors. The complex interplay of physical, chemical, biological and ecological constraints does not guarantee that absolute optima are achieved by the evolutionary process, and makes the design of optimal organisms hard to predict from first principles [1, $2,3]$. The evolutionary dynamics itself introduces further complications: for instance, finite populations can accumulate deleterious mutations [4], and species which are not the fittest may nevertheless prevail if they are more tolerant to mutations (survival of the flattest vs. survival of the fittest) [5]. Moreover, since no organism can be optimal in every environment, tradeoffs are imposed on the development and maintenance of any structure and function in living systems.

Microbes are ideal experimental systems to develop and test theories about optimality, tradeoffs and constraints in biology. The relative ease in manipulating microbial genomes and fast reproduction rates allows experimentalists to generate mutants with the desired features, to measure their costs and benefits, and to follow the evolutionary dynamics for thousands of generations [6].

\footnotetext{
(C) 2012 Elsevier Ltd. All rights reserved.

Corresponding Author: Andrea Velenich, 77 Massachusetts Avenue, 13-2054, Cambridge, MA, USA, Tel: +1 617-715-4326, Fax: +1 617-258-6883, velenich@mit.edu.

Publisher's Disclaimer: This is a PDF file of an unedited manuscript that has been accepted for publication. As a service to our customers we are providing this early version of the manuscript. The manuscript will undergo copyediting, typesetting, and review of the resulting proof before it is published in its final citable form. Please note that during the production process errors may be discovered which could affect the content, and all legal disclaimers that apply to the journal pertain.
} 


\section{Metabolic constraints}

The common observation that the growth rate of a cell is inversely correlated with its resistance to stress [7] suggests the existence of a trade-off between growth and repair. More broadly, the growth rate may affect the entire economy of a cell through system-level constraints. An example of this effect is the up to 10-fold variations in the production rates of constitutively expressed genes during different growth phases [8]. Eventually, nonlinearities in the growth-rate/gene-expression feedback may even lead to bistable growth [9] and possibly cause some cells to enter a dormant state [8]. Growth-rate dependent global constraints have recently been expressed as simple linear relations involving the expression levels of different classes of proteins (ribosomal, growth-rate dependent, growth-rate independent and unnecessary) $[10,11,12]$.

The macroscopic description of metabolism based on growth rates is complemented by the detailed study of metabolic constraints and the optimization of metabolic fluxes to maximize biomass production $[13,14]$. These methods have been formalized into flux balance models describing all the chemical reactions in a cell [15]. Even more comprehensive models account also for the costs and benefits of enzyme synthesis [16] to describe, for instance, the switch between efficient respiration in nutrient-poor environments and inefficient fermentation in nutrient-rich environments [17].

\section{Trade-offs and gene regulation}

One of the primary roles of gene regulation in microbes is to modulate metabolic fluxes and to ensure that high growth rates can be achieved in favorable environmental conditions. A common synthetic technique to characterize cellular responses is then to decouple a response in gene expression from its triggering signals.

Poelwijk et al. [18] cleverly improved on previous experiments [19] measuring the costs and benefits of the lac system in $E$. coli by decoupling induction and metabolic activity. The cost of the lac operon expression has been measured by tuning protein synthesis with an inducer which cannot be metabolized, whereas the benefits of expression have been measured with a metabolite which cannot induce. Evolutionary experiments in environments containing different concentrations of inducer and metabolite have confirmed how a theoretical costs/ benefits analysis can predict the result of adaptation in each environment.

Similarly, by decoupling glucose sensing and glucose uptake in yeast, it has been shown how the growth rate of yeast cells depends separately on these two variables. This is because sensing high concentrations of extracellular glucose triggers the synthesis of extra glucoseprocessing machinery and imposes a fitness burden if glucose import is less than expected [20].

When a regulatory system is not present, it can be engineered using synthetic techniques. As an example, in yeast, low levels of noise in the expression of a glutamate synthase provide a fitness advantage in limiting concentrations of ammonia, whereas noisy expression increases fitness at toxic levels of ammonia. A mutant with the ability to tune noise in response to a signaling molecule has been built, and its evolutionary advantage in fluctuating environments over its non-regulated wild type competitors has been established [21].

\section{Fitness landscapes and evolutionary accessibility}

Gene regulation confers a fitness advantage when the time scale of the environmental fluctuation is shorter than a few hundred generations [22, 23]. If the environment changes much more slowly, however, regulatory functions are typically lost, according to a "use it or 
lose it" principle $[24,25]$ and the optimal expression level is fixed through genetic mutations. An example of this transition between regulation on cellular time-scales to adaptation on evolutionary time-scales has been repeatably observed in laboratory populations of yeast cells: when a selective pressure favors fast growth, a trade-off between growth rate and mating efficiency causes quick silencing and an eventual loss of 23 genes in the mating pathway to achieve a $2 \%$ growth rate advantage [26].

Fitness landscapes assign a fitness value to each genotype or phenotype (in a specified environment) and they represent a geometric setting in which to study the evolutionary process connecting a low-fitness ancestral organism to its high-fitness descendants (Figure 1a). Whereas evolution can proceed rapidly along adaptive paths of increasingly fit organisms [27, 28, 29], real populations do cross moderately shallow fitness valleys under a variety of conditions: if the fitness deficit of the intermediates is small enough [30], if the mutation rate is large enough, if the available time is long enough or by exploiting small effective population sizes (e.g. at the frontier of a range expansion [31]).

Synthetic approaches are increasingly applied to delineate naturally occurring landscapes by constructing all the possible genetic intermediates between ancestral organisms and their evolved counterparts $[27,28,32,33,34,35,36,37,38]$. Although such experimental studies are necessarily limited by the exponential number of possible intermediates, various kinds of landscapes have been studied. Notably, the mapping involved in the definition of a landscape is not necessarily one-to-one: different genotypes can converge to the same phenotype [39] and, in case of multi-stable gene expression [40], the same genotype can produce different phenotypes. Moreover, even if genotype-to-phenotype landscapes may be approximately linear, the non-linearity of the further mapping between phenotype and growth rate leads to a non-linearity of the final genotype-to-fitness landscapes [32], which often display epistatic effects leading to diminishing returns [33, 34, 35].

Starting from the geometric properties of experimentally measured landscapes, the properties of the evolutionary dynamics can be predicted or, vice versa, some properties of naturally occurring fitness landscapes can be inferred by observing the evolutionary dynamics. Especially relevant properties of fitness landscapes are the ones which do not depend on how phenotypes or growth rates are scaled. Examples of these topological features are the number of accessible paths leading to a fitness maximum, the number of peaks in a landscape, and the presence of sign epistasis (Figure 1b), a necessary condition for the generation of multi-peaked landscapes [41]. Sign epistasis has been further shown to reduce the number of accessible evolutionary paths [42], to increase their length by imposing genetic detours [43], to increase the repeatability of evolution [44], and to interfere with its reversibility $[36,45]$.

Notably, the biochemical constraints encoded by sign epistasis can also be exploited to encode biological functions. This is highlighted in a landscape describing the activity of a lac promoter as a function of mutations in the lac repressor and in its operator [37]. Each repressor/operator pair can be interpreted as a key-lock system and the fact that the landscape is multi-peaked (due to sign epistasis) indicates that a different key-lock system [46] can be generated with a few mutations in the repressor and a few compensatory mutations in the operator.

Novel regulatory functions can indeed evolve with surprising rapidity [38, 47]. In [38], a lac promoter controlled a synthetic operon for the simultaneous expression of two enzymes in a laboratory population of E. coli. High expression levels of the operon conferred higher fitness in environment $\mathrm{A}$ and low fitness in environment $\mathrm{B}$. While alternating between the two environments, the experimenters added an inducer molecule only in environment $B$, so 
that the operon was highly expressed when low expression would have been optimal and repressed when high expression would have been optimal. After only three rounds of mutagenesis and competitive growth, the logic of regulation in the bacterial population was reversed, so that the inducer molecule had effectively become an inhibitor. Surprisingly, only three key mutations in the lac repressor protein were necessary to develop this new mode of regulation $[38,48]$.

\section{Synthetic ecosystems}

Natural extensions of the formalism used to describe constraints and trade-offs at the singlecell level (Figure 2a) have recently been applied to small synthetic ecosystems, where each species can be considered as a module with specific metabolic inputs and outputs [49].

As a simple proof of principle, two variants of a yeast strain have been built, each overproducing an amino acid essential for the other strain (Figure 2b). In an environment without the two amino acids the two strains were obligate cooperators [50] and, in some conditions, they managed to coexist. In a similar experiment with $E$. coli, all possible pairing of 46 auxotrophic strains have been explored. In $\sim 17 \%$ of the cases, the two strains were able to provide each others' essential nutrients and survive in environments which would have killed each of them separately [51].

Stable cooperation in synthetic ecosystems which did not co-evolve is not guaranteed. In fact, in well-mixed conditions, asymmetries in growth rates, different tolerance to insults such as starvation or antibiotics and delays in the release of metabolites often result in the rapid rise of one strain, followed by the collapse of the whole population [52]. However, since these mutualistic interactions are based on the exchange of diffusible molecules, spatially structured environments (typically with length scales in the hundreds of microns) may enable the existence of synthetically assembled multi-species microbial communities [52].

Alternatively, even in spatially unstructured environments, the temporal structure of a population can play a relevant role in the coexistence of multiple species. Starting from the same ancestral $E$. coli strain, the seasonality induced by daily serial dilutions promoted the co-evolution of two strains, each one occupying its own temporal niche: early in the day a fast-growing strain feasted on fresh medium; later in the day a scavenger strain thrived by cannibalizing the first strain [53].

Due to the complexity of even the smallest laboratory ecosystems discussed above, microbial communities are a standing challenge for synthetic and systems biology. Notably, about $1 / 3$ of the genes in $E$. coli still have no known function and a fraction of those genes might be related to the microbes' social life. Even more strikingly, the majority of microbial species cannot be cultured in isolation and much of the uncharted territory in the microbial world might remain unexplored until the networks of ties and constraints in microbial communities are understood [54].

\section{The spread of cooperation}

In microbial consortia each organism follows a selfish strategy, exploiting other organisms, preying on them [55] or synthesizing toxins for chemical warfare [56]. However, cooperative and altruistic behaviors are often observed in nature, in apparent contrast with the evolution of selfish genes. The emergence and, even more importantly, the persistence of cooperative behaviors can be investigated using synthetic techniques which allow scientists to manipulate microbial strategies. Communities of engineered microbes become then a tool to understand ecosystems beyond the microbiology laboratory. 
Yeast cells grown on sucrose provide a model system to study cooperative behaviors [57, 58]. Cooperator cells pay an individual cost to hydrolyze sucrose, producing glucose as a common good which diffuses in the medium (Figure 2c). Cheater cells, instead, are engineered to take advantage of the glucose in the medium without paying the cost for its production. Cheater cells can readily invade a population of cooperators and spread.

Surprisingly, however, even in a well-mixed environment it has been shown that coexistence is possible [57]. Although cooperators are always exploited by cheaters, cooperators can survive by having preferential access to the common good they produce, and fare relatively well when the common good is scarce. Spatially structured populations further promote cooperation $[59,60]$ : since a common good typically diffuse in the environment, local subcommunities which happen to be rich in cooperators also become privileged recipients of the common good and grow faster, so that the overall fraction of cooperators increases. This is the essence of the Simpson's paradox, which recent experiments on engineered $E$. coli have explicitly demonstrated [61]. To further emphasize the importance of laboratory models for a thorough understanding of natural ecosystems, Chuang et al. [62] used a simple bacterial system to demonstrate that costs and benefits can be non-linear functions of the population structure, and that costs and benefits measured at the cell level are not simply related to the same parameters for the whole population.

\section{Conclusion}

We briefly reviewed recent experiments and theoretical work to understand the structure of metabolic, regulatory, evolutionary and social constraints. Despite considerable effort, these pioneering studies are partial or isolated results, providing mostly anecdotal evidence. Encouragingly, there is a trend of ever cheaper and more widespread use of synthetic biology tools to read and write genomes, and of biophysics techniques to quantify the behavior of living cells. This trend is enabling the acquisition of an unprecedented amount of data [63] and of aggressive hypothesis testing. Independent studies on a variety of systems are rapidly accumulating: the generic features of fitness landscapes [64] will reveal the constraints of evolution as a stochastic optimization process, whereas the emerging motifs in the networks of interactions within microbial communities will soon have a direct impact on biotechnology and human health.

\section{Acknowledgments}

The Gore laboratory gratefully acknowledges the financial support of a Sloan Foundation Fellowship, the Pew Scholars Program, an NSF CAREER Award, and an NIH R00 Pathways to Independence Award.

\section{References and recommended reading}

1. Pérez-Escudero A, Rivera-Alba M, de Polavieja GG. Structure of deviation from optimality in biological systems. Proc Natl Acad Sci. 2009; 106:20544-20549. [PubMed: 19918070]

2. Gutenkunst RN, Waterfall JJ, Casey FP, Brown KS, Myers CR, Sethna JP. Universally sloppy parameters sensitivities in systems biology models. PLoS Computational Biology. 2007; 3:e189.

3. Daniels BC, Chen YJ, Sethna JP, Gutenkrust RN, Myers CR. Sloppiness, robustness and evolvability in systems biology. Curr Opin Biotechnol. 2008; 19:389-395. [PubMed: 18620054]

4. Fernández A, Lynch M. Non-adaptive origins of interactome complexity. Nature. 2011; 474:502505. [PubMed: 21593762]

5. Beardmore RE, Gudelj I, Lipson DA, Hurst LD. Metabolic trade-offs and the maintenance of the fittest and the flattest. Nature. 2011; 472:342-346. [PubMed: 21441905]

6. Barrick JE, Yu DS, Yoon SH, Jeong H, Oh TK, Schneider D, Lenski RE, Kim JF. Genome evolution and adaptation in a long-term experiment with Escherichia coli. Nature. 2009; 461:12431247. [PubMed: 19838166] 
7. López-Maury L, Marguerat S, Bähler J. Tuning gene expression to changing environments. from rapid responses to evolutionary adaptation. Nat Rev Genet. 2008; 9:583-593. [PubMed: 18591982]

8. Klumpp S, Zhang Z, Hwa T. Growth rate-dependent global effects on gene expression in bacteria. Cell. 2009; 139:1366-1375. [PubMed: 20064380]

9. Tan C, Marguet P, You L. Emergent bistability by a growth-modulating positive feedback circuit. Nat Chem Biol. 2009; 11:842-848. [PubMed: 19801994]

**10. Scott M, Gunderson CW, Mateescu EM, Zhang Z, Hwa T. Interdependence of cell growth and gene expression: origins and consequences. Science. 2010; 330:1099-1102. Develops simple phenomenological laws describing the global constraints between growth-rate, cellular composition and protein expression. [PubMed: 21097934]

11. Scott M, Hwa T. Bacterial growth laws and their applications. Curr Opin Biotechnol. 2011; 22:559-565. [PubMed: 21592775]

12. Shachrai I, Zaslaver A, Alon U, Dekel E. Cost of unneeded proteins in E. coli is reduced after several generations in exponential growth. Molecular Cell. 2010; 38:758-767. [PubMed: 20434381]

13. Edwards JS, Ibarra RU, Palsson BO. In silico predictions of Escherichia coli metabolic capabilities are consistent with experimental data. Nat Biotechnol. 2001; 19:125-130. [PubMed: 11175725]

14. Ibarra RU, Edwards JS, Palsson BO. Escherichia coli K-12 undergoes adaptive evolution to achieve in silico predicted optimal growth. Nature. 2002; 420:186-189. [PubMed: 12432395]

15. Orth JD, Thiele I, Palsson BO. What is flux balance analysis? Nat Biotechnol. 2010; 28:245-248. [PubMed: 20212490]

16. Stoebel DM, Dean AM, Dykhuizen DE. The cost of expression of Escherichia coli lac operon proteins is in the process, not in the products. Genetics. 2008; 178:1653-1660. [PubMed: 18245823]

17. Molenaar D, van Berlo R, de Ridder D, Teusink B. Shifts in growth strategies reflect tradeoffs in cellular economics. Mol Sys Biol. 2009; 5:323.

**18. Poelwijk FJ, Heyning PD, de Vos MGJ, Kiviet DJ, Tans SJ. Optimality and evolution of transcriptionally regulated gene expression. BMC Systems Biology. 2011; 5:128. Provides a direct measurement of the benefits and costs of gene expression by decoupling regulation and metabolic function. It also demonstrates how evolution rapidly tunes gene regulation to achieve optimal expression. [PubMed: 21846366]

19. Dekel E, Alon U. Optimality and evolutionary tuning of the expression level of a protein. Nature. 2005; 436:588-592. [PubMed: 16049495]

20. Youk H, van Oudenaarden A. Growth landscape formed by perception and import of glucose in yeast. Nature. 2009; 462:875-879. [PubMed: 20016593]

21. Bayer TS, Hoff KG, Beisel CL, Lee JJ, Smolke CD. Synthetic control of a fitness tradeoff in yeast nitrogen metabolism. Journal of Biological Engineering. 2009; 3:1. [PubMed: 19118500]

22. Kussell E, Leibler S. Phenotypic diversity, population growth, and information in fluctuating environments. Science. 2005; 309:2075-2078. [PubMed: 16123265]

23. Munstonen V, Lässig M. From fitness landscapes to seascapes: non-equilibrium dynamics of selection and adaptation. Trends in Genetics. 2009; 25:111-119. [PubMed: 19232770]

24. Gerland U, Hwa T. Evolutionary selection between alternative modes of gene regulation. Proc Natl Acad Sci. 2009; 106:8841-8846. [PubMed: 19470486]

25. McCutcheon JP, Moran N. Extreme genome reduction in symbiotic bacteria. Nat Rev Microbiol. 2012; 10:13-26. [PubMed: 22064560]

26. Lang GI, Murray AW, Botstein D. The cost of gene expression underlies a fitness trade-off in yeast. Proc Natl Acad Sci. 2009; 106:5755-5760. [PubMed: 19299502]

27. Weinreich DM, Delaney NF, DePristo MA, Hartl DL. Darwinian evolution can follow only very few mutational paths to fitter proteins. Science. 2006; 312:111-114. [PubMed: 16601193]

28. Poelwijk FJ, Kiviet DJ, Weinreich DM, Tans SJ. Empirical fitness landscapes reveal accessible evolutionary paths. Nature. 2007; 445:383-386. [PubMed: 17251971]

29. Franke J, Klözer A, de Visser AGM, Krug J. Evolutionary accessibility of mutational pathways. PloS Comput Biol. 2011; 7:e1002134. [PubMed: 21876664] 
30. Weissman DB, Desai MM, Fisher DS, Feldman MW. The rate at which asexual populations cross fitness valleys. Theor Popul Biol. 2009; 75:286-300. [PubMed: 19285994]

31. Burton OJ, Travis JMJ. The frequency of fitness peak shifts is increased at expanding range margins due to mutation surfing. Genetics. 2008; 179:941-950. [PubMed: 18505864]

32. Lunzer M, Miller SP, Felsheim R, Dean AM. The biochemical architecture of an ancient adaptive landscape. Science. 2005; 310:499-501. [PubMed: 16239478]

*33. Khan AI, Dinh DM, Schneider D, Lenski RE, Cooper TF. Negative epistasis between beneficial mutations in an evolving bacterial population. Science. 2011; 332:1193-1196. Reconstructs a fitness landscapes from five beneficial mutations fixed during adaptation and determines the prevalence of negative epistasis among such mutations. [PubMed: 21636772]

*34. Chou HH, Chiu HC, Delaney NF, Segrè D, Marx CJ. Diminishing returns epistasis among beneficial mutations decelerates adaptation. Science. 2011; 332:1190-1192. Reconstructs a fitness landscapes from four beneficial mutations fixed during adaptation and establishes a principle of diminishing returns, by which the beneficial fitness effect of a mutation is smaller when it appears on a fitter background. [PubMed: 21636771]

35. Kvitek DJ, Sherlock G. Reciprocal sign epistasis between frequently experimentally evolved adaptive mutations causes a rugged fitness landscape. PLoS Genetics. 2011; 7:e1002056. [PubMed: 21552329]

36. Tan L, Serene S, Chao HX, Gore J. Hidden Randomness Between Fitness Landscapes Limits Reverse Evolution. Phys Rev Lett. 2011; 106:198102. [PubMed: 21668204]

*37. Dawid A, Kiviet DJ, Kogenaru M, de Vos M, Tans SJ. Multiple peaks and reciprocal sign epistasis in an empirically determined genotype-phenotype landscape. Chaos. 2010; 20:026105. Provides experimental evidence of a naturally occurring multi-peaked fitness landscape and discusses its biological relevance in encoding distinct repressor/operator pairs. [PubMed: 20590334]

**38. Poelwijk FJ, de Vos MGJ, Tans SJ. Tradeoffs and optimality in the evolution of gene regulation. Cell. 2011; 146:462-470. Provides an experimental demonstration of how the logic of a regulatory circuit can be rapidly reversed by evolution once a key mutation (representing a bottleneck in the fitness landscape) is acquired. It also shows how a quantitative understanding of trade-offs in fluctuating environments leads to correct predictions of the evolutionary dynamics. [PubMed: 21802129]

39. Sabarly V, Bouvet O, Glodt J, Clermont O, Skurnik D, Diancourt L, de Vienne D, Denamur E, Dillmann C. The decoupling between genetic structure and metabolic phenotypes in Escherichia coli leads to continuous phenotypic diversity. J Evol Biol. 2011; 24:1559-1571. [PubMed: 21569155]

*40. Perfeito L, Ghozzi S, Berg J, Schnetz K, Lässig M. Nonlinear fitness landscape of a molecular pathway. PLoS Genetics. 2011; 7:e1002160. Shows how feedback loops involving the growth rate produce bi-stability in the fitness landscape and generate cliffs which constrain evolution. [PubMed: 21814515]

41. Poelwijk FJ, Tănase-Nicola S, Kiviet DJ, Tans SJ. Reciprocal sign epistasis is a necessary condition for multi-peaked fitness landscapes. J Theor Biol. 2011; 272:141-144. [PubMed: 21167837]

42. Povolotskaya IS, Kondrashov FA. Sequence space and the ongoing expansion of the protein universe. Nature. 2010; 465:922-926. [PubMed: 20485343]

43. DePristo MA, Hartl DL, Weinreich DM. Mutational reversions during adaptive protein evolution. Mol Biol Evol. 2007; 24:1608-1610. [PubMed: 17556755]

44. Toprak E, Veres A, Michel JB, Chait R, Hartl DL, Kishony R. Evolutionary paths to antibiotic resistance under dynamically sustained drug selection. Nat Genet. 2012; 44:101-105. [PubMed: 22179135]

45. Bridgham JT, Ortlund EA, Thornton JW. An epistatic ratchet constrains the direction of glucocorticoid receptor evolution. Nature. 2009; 461:515-519. [PubMed: 19779450]

46. Eldar A. Social conflict drives the evolutionary divergence of quorum sensing. Proc Natl Acad Sci. 2011; 108:13635-13640. [PubMed: 21807995] 
47. Marx CJ. Regulatory revolution: evolving the "anti-lacl” repressor. Cell. 2011; 146:350-352. [PubMed: 21816271]

48. Mayo AE, Setty Y, Shavit S, Zaslaver A, Alon U. Plasticity of the cis-regulatory input function of a gene. PLoS Biology. 2006; 4:e45. [PubMed: 16602820]

49. Klitgord N, Segrè D. Ecosystems biology of microbial metabolism. Curr Opin Biotechnol. 2011; 22:1-6. [PubMed: 21190838]

50. Shou W, Ram S, Vilar JMG. Synthetic cooperation in engineered yeast populations. Proc Natl Acad Sci. 2007; 104:1877-1882. [PubMed: 17267602]

51. Wintermute EH, Silver PA. Emergent cooperation in microbial metabolism. Mol Sys Biol. 2010; 6:407.

52. Kim HJ, Boedicker JQ, Choi JW, Ismagilov RF. Defined spatial structure stabilizes a synthetic multispecies bacterial community. Proc Natl Acad Sci. 2008; 105:18188-18193. [PubMed: 19011107]

53. Rozen RE, Philippe N, de Visser JA, Lenski RE, Schneider D. Death and cannibalism is a seasonal environment facilitate bacterial coexistence. Ecology Letters. 2008; 12:34-44. [PubMed: 19019196]

54. Wintermute EH, Silver PA. Dynamics in the mixed microbial concourse. Genes Dev. 2010; 24:2603-2614. [PubMed: 21123647]

55. Balagaddé FK, Song H, Ozaki J, Collins CH, Barnet M, Arnold FH, Quake SR, You L. A synthetic Escherichia coli predator-prey ecosystem. Mol Sys Biol. 2008; 4:187.

56. Majeed H, Gillor O, Kerr B, Riley MA. Competitive interactions in Escherichia coli populations: the role of bacteriocins. ISME J. 2011; 5:71-81. [PubMed: 20664553]

57. Gore J, Youk H, van Oudenaarden A. Snowdrift game dynamics and facultative cheating in yeast. Nature. 2009; 459:253-256. [PubMed: 19349960]

58. Greig D, Travisano M. The prisoner's dilemma and polymorphism in yeast SUC genes. Proc Biol Sci. 2004; 271(suppl 3):S25-S26. [PubMed: 15101409]

59. MacLean RC, Fuentes-Hernandez A, Greig D, Hurst LD, Gudelj I. A mixture of "cheats" and "cooperators" can enable maximal group benefit. PloS Biol. 2010; 8:e1000486. [PubMed: 20856906]

60. Koschwanez JH, Foster KR, Murray AW. Sucrose Utilization in Budding Yeast as a Model for the Origin of Undifferentiated Multicellularity. PLoS Biol. 2011; 9:e1001122. [PubMed: 21857801]

61. Chuang JS, Rivoire O, Leibler S. Simpson's paradox in a synthetic microbial system. Science. 2009; 323:272-275. [PubMed: 19131632]

*62. Chuang JS, Rivoire O, Leibler S. Cooperation and Hamilton's rule in a simple synthetic microbial system. Mol Sys Biol. 2010; 6:398. Applies synthetic biology to tune the costs and benefits of an altruistic behavior in E. coli and exploits such a controlled system to highlight the mathematical subtleties involved in the maintenance of altruism.

63. Costanzo M, Baryshnikova A, Bellay J, Kim Y, Spear ED, Sevier CS, Ding H, Koh JLY, Toufighi K, Mostafavi S, et al. The genetic landscape of a cell. Science. 2010; 327:425-431. [PubMed: 20093466]

64. Szendro, IG.; Schenk, MF.; Franke, J.; Krug, J.; de Visser, JAGM. Quantitative analyses of empirical fitness landscapes. arXiv:1202.4378 


\section{Highlights}

- biological constraints are apparent at different scales, from cells to ecosystems

- empirical relations involving growth rates reveal intra-cellular constraints

- variable environments impose trade-offs and select for optimal gene regulation

- rugged fitness landscapes constrain the evolutionary process and its outcome

- stable ecosystems are constrained by their environmental and social structures 


\section{a}

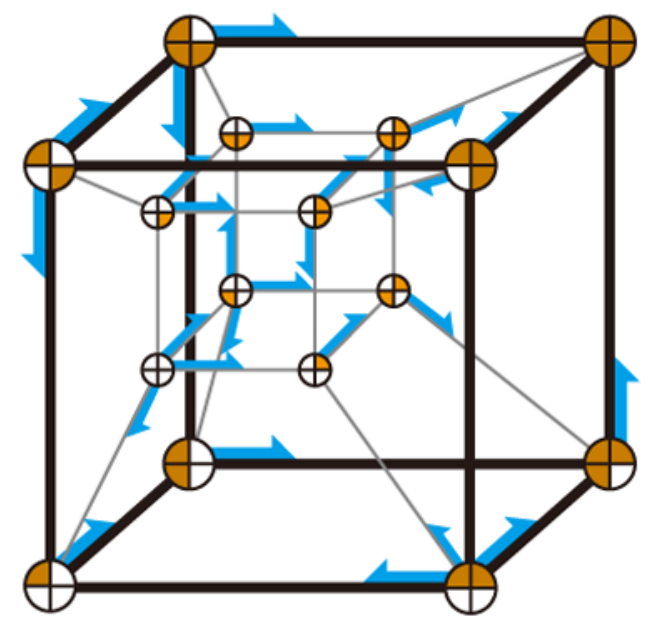

b

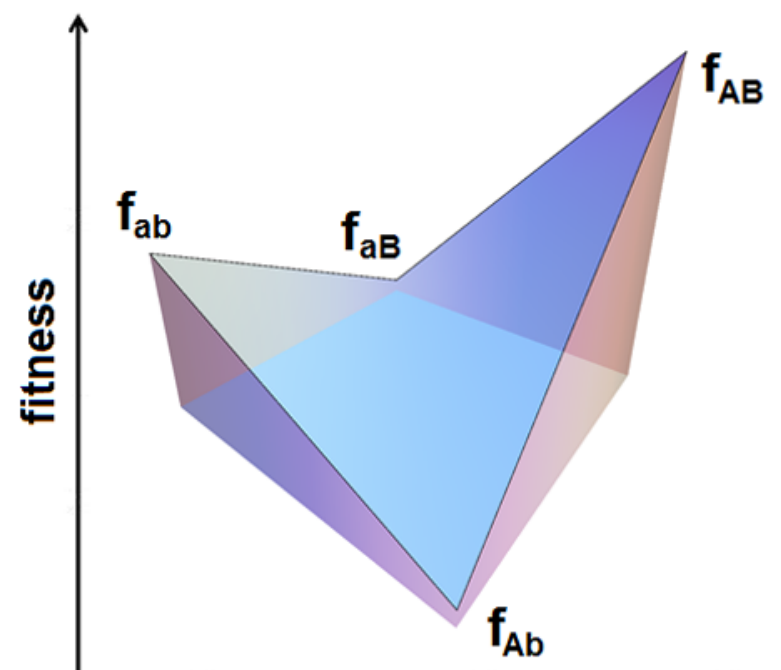

genotypes

Figure 1.

The geometry of fitness landscapes. a Example of an experimentally mapped 4-dimensional phenotypic landscape describing the effectiveness (measured as MIC, Minimum Inhibitory Concentration) of a beta-lactamase enzyme in hydrolysing the antibiotic cefotaxime [27, 36]. Every circle represents a genotype for which each of the four mutations can be absent (white wedge) or present (orange wedge). The blue arrows indicate the selective pressure to acquire mutations which increase the MIC. The landscape in this example is rugged, meaning that some mutations can be beneficial or deleterious depending on the genetic background in which they appear. The landscape is nevertheless single-peaked. b An example of sign epistasis on a 2-dimensional landscape. Whereas starting from a wild type genotype $(\mathrm{ab})$ the combined fitness effect of two mutations (AB) is beneficial, the effect of only one of the two mutations (either A or B) is deleterious. For this reason the evolutionary dynamics is not guaranteed to find the global maximum of fitness $\left(\mathrm{f}_{\mathrm{AB}}\right)$ and it might remain stuck for long times in a sub-optimal local maximum $\left(f_{a b}\right)$. 
a

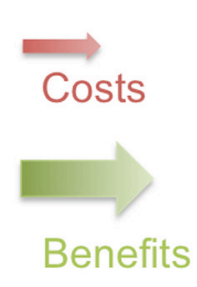

Self-serving metabolism

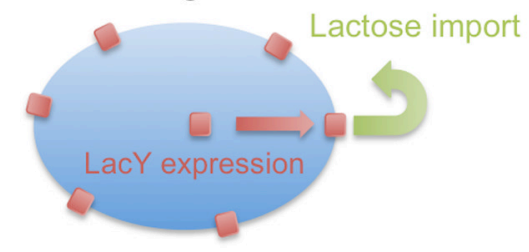

b

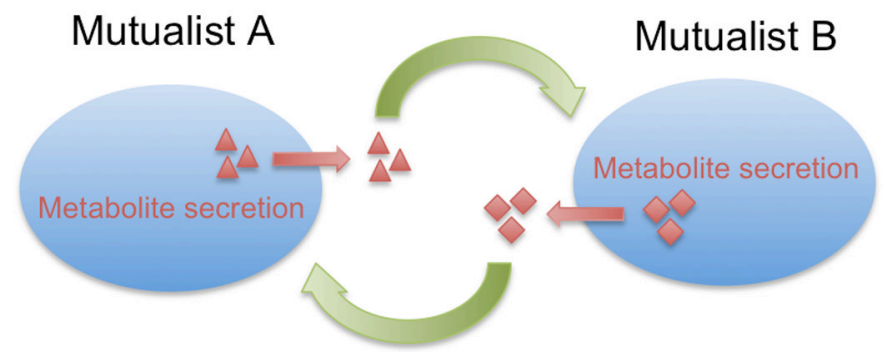

Metabolite diffusion

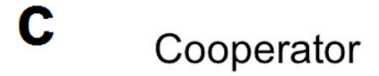

Cheater
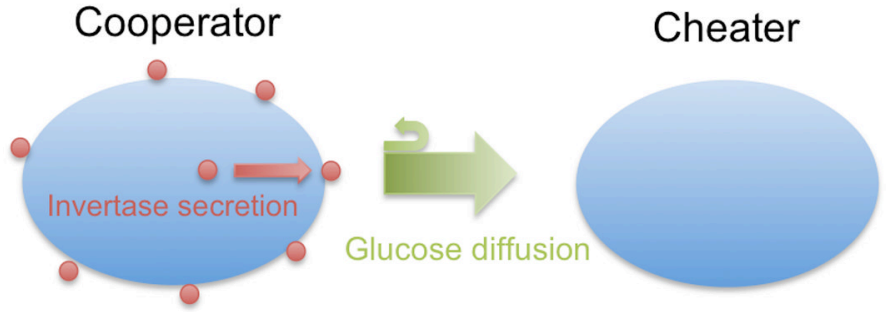

Figure 2.

Cost/benefit analyses at multiple scales. a Single cells quickly adapt to balance the costs associated with the expression of a gene and the benefits deriving from the gene products in order to achieve optimal growth rates [18]. b Bacterial communities are often based on mutualistic interactions. A-cells might pay a cost so that B-cells can benefit from it. In turn, A-cells indirectly benefit from the success of B-cells. c Despite the existence of cells which do not cooperate, cooperative behaviors can still be maintained [57] if cooperator cells have preferential access to a public good (e.g. if a diffusive dynamics ensures that producers are also exposed to higher concentrations of the common good). Quantitative details of the costs and benefits, together with the spatial [52] and temporal [50, 53, 55] structures of the interactions are often crucial for the evolutionary stability of these social interactions. 